\title{
A Diesel Engine Model for Dynamic Drive Cycle Simulations
}

\author{
Markus Grahn* Jan-Ola Olsson* Tomas McKelvey** \\ * Volvo Car Corporation, Gothenburg, Sweden, (e-mail: mgrahn1, \\ jolsso56@volvocars.com). \\ ** Department of Signals and Systems, Chalmers University of \\ Technology, SE-412 96, Gothenburg, Sweden (e-mail: \\ tomas.mckelvey@chalmers.se)
}

\begin{abstract}
The development and implementation of a diesel engine combustion system simulation model is described. The model is a crank angle based combustion model, which uses the conditions in the intake and exhaust manifolds together with the fuel injection signal from the engine control unit to estimate the in-cylinder pressure throughout a complete combustion cycle. The model is implemented in Matlab. Furthermore, a Simulink coupling has been developed and implemented such that the combustion model can be connected directly to a Simulink mean value model of an engine air system. The coupling makes the combustion model act like a continuous source and a continuous sink in a mean value model. The coupling makes it possible to continuously simulate an engine in steady-state or transient operation, while the combustion model produces estimated cylinder pressure traces for each combustion cycle. This makes it possible to estimate fuel consumption and to couple the model with emission models which use the cylinder pressure or the rate of heat release as input. The model is developed, calibrated and verified using measured data from a 2.4 liter Volvo diesel engine, equipped with a turbocharger, an exhaust gas recirculation system, and a common rail injection system. The combustion model estimates $I M E P_{n e t}$ with a correlation factor of 0.995 for the used data. The simulation time is in the range between 1 and 25 milliseconds for one combustion cycle on a standard computer, depending on the implementation.
\end{abstract}

Keywords: Diesel engine modeling, Diesel engine control, Engine control optimization.

\section{INTRODUCTION}

Modern passenger car diesel engines become increasingly complex in order to meet stricter law requirements on emissions and stronger demand for lower fuel consumption. To be able to handle these requirements, more and more controllable systems are added, leading to more degrees of freedom for the engine operation. Examples of this in a typical passenger car diesel engine today are controllable boost pressure, controllable exhaust gas recirculation rate, controllable fuel rail pressure, multiple injections with controllable timings and durations. This opens up for the possibility to operate the engine at more efficient conditions, but it also increases the complexity to optimize the calibration of the Engine Management System (EMS), i.e. to find control strategies and set points which lead to optimal operation of the combustion system.

To efficiently address this complex design task a model based design strategy is a viable complement to more classical tests directly on the engine. Initial EMS design and optimization can be performed using simulation techniques utilizing a model of the relevant engine system and reduce the need of extensive test of the physical engine in the test facility. A simulation based approach also can be employed in early design phases where different designs can be simulated and the results evaluated before building a physical engine. Using this approach there is an obvious trade-off between model accuracy and simulation speed. The model accuracy need to be good enough such that the optimized engine calibration for the simulation model also is valid for the real engine, but to be able to perform the optimization within a reasonable time, the simulation models also need to be as fast as possible to execute.

Several types of engine simulation models exist ranging from detailed CFD calculation (Shrivastava et al. (2002)) of the combustion process to purely data-driven map based models (Brahma and Rutland (2003)). The very detailed models typically suffer from long execution time, making them less suitable for EMS optimization. The pure data-driven models are used for EMS optimization today (Atkinson et al. (2008); Brahma et al. (2009)), but are very sensitive for small changes in the air system, e.g. a resized turbocharger. Semi-empirical models for the combustion have also been used for EMS optimization (Arsie et al. (2007)), however limited to steady-state engine operation.

This paper describes the implementation of a simulation model suitable for dynamic drive cycle simulations. The model is composed of a diesel engine combustion model coupled with a mean value model for the air system. These models together with a model for the EMS and models for a vehicle and a driver make it possible to simulate a dynamic vehicle driving cycle for a specific calibration of the engine control unit, and to receive cylinder pressure es- 
timations for all combustions throughout the driving cycle. By utilizing the cylinder pressure estimation together with other engine states and emission models such as Andersson et al. (2006); Kirchen and Boulouchos (2009), the emissions for each combustion in the cycle can be predicted. Hence, the fuel consumption and the accumulated engine out emissions for a complete dynamic vehicle driving cycle can be predicted. Using the simulation models and adding an optimization strategy, EMS calibration settings can be found which minimize fuel consumption while emissions are kept within required limits.

\subsection{Modelling approach}

A dynamic driving cycle includes both stationary and transient engine operating conditions. In order to be able to predict how emissions and fuel consumption depend on a given EMS calibration, the model must be able to resolve the dynamic effects. For example the model must capture the boost pressure build up during vehicle acceleration and how the resulting varying conditions in the inlet manifold is manifested in the engine out emissions. This observation leads us to divide the model into two parts. First, a physical based mean value air system model including volumes, orifices, valves and other air system components, where the cylinders acts as an air sink for the inlet manifold and an air source for the exhaust manifold. This model will provide a time resolved state of the inlet air composition in both amount of species as well as pressure and temperature. And second, a model for the combustions, which are modelled as discrete events at times controlled by the engine speed. The gas mixture information from the air model together with a simple heat-release model modulated by the injection phasing and timing are used to model the crank resolved pressure in the cylinder. The gas states at the exhaust valve opening are then used as boundary conditions for the exhaust part of the air system model, thus closing the air-combustion model loop.

\subsection{Engine measurement data}

To develop, calibrate and verify the model, measurement data from a 5-cylinder Volvo diesel engine was used. The engine is equipped with a common-rail injection system, a turbocharger with variable geometry, charge air cooling, an exhaust gas recirculation (EGR) system with cooling, and has a displacement volume of 2.4 litres.

3713 steady-state measurements were performed on the engine in the complete speed and load operating area, ranging from 750 to $4750 \mathrm{rpm}$ and from 0 to 24 bar IMEP. For each speed/load operating point, several measurements were performed. For each measurement, the nominal setting of the fuel rail pressure and the injection strategy, including fuel masses and dwell times were used. However, the timing of the injection package, the boost pressure, and the exhaust gas recirculation rate were varied around the nominal settings. Depending on the engine operating point, up to four injections were used, two pilot injections, one main injection, and one post injection. From this data, 3342 random measurements were used for the calibration of the model, and the remaining 371 measurements were used for verification. The engine was equipped with sensors such that the pressure and temperature in the intake manifold, the pressure and temperature in the exhaust manifold, the fresh air mass flow, and the exhaust gas recirculation mass flow could be measured. The engine was also equipped with in-cylinder pressure sensors. From the EMS, the engine speed, the crank angle degrees (CAD) for start and end of injection, and the injected fuel mass for each injection were registered.

\section{ENGINE MODELING}

This section describes the two parts of the engine model; the crank angle resolved combustion model, and the coupling with a mean value model of an engine air system.

\subsection{Combustion model}

To be able to model the combustion process, the conditions in the cylinder at intake valve closing (ivc), i.e. the pressure, the total mass of different species, the temperature, and the cylinder volume, must be estimated. These estimations should be based on states that could be given by a mean value air system model for an engine, i.e. the pressure and temperature in the intake manifold, the pressure in the exhaust manifold, and the mass flow of different species into the engine. The pressure in the cylinder at ivc is calculated from the pressure in the intake manifold. Before the intake valves are fully closed, there is some compression within the cylinder, dependent on the timing of the intake valve closing and the intake valve lift profile. The pressure in the cylinder at ivc is modelled as:

$$
p_{\text {ivc }}=C_{p_{\text {ivc }}} p_{\text {intake }}
$$

where:

$$
\begin{array}{ll}
p_{i v c} & \text { is the cylinder pressure at intake valve clos- } \\
& \text { ing }(\mathrm{Pa}) \\
p_{\text {intake }} & \text { is the pressure in the intake manifold }(\mathrm{Pa}) \\
C_{p_{\text {ivc }}} & \text { is an empirical constant }(-)
\end{array}
$$

The constant gain, $C_{p_{i v c}}$, is calibrated such that the difference between the modelled pressure and the pressure from the in-cylinder pressure sensor at ivc is minimized in a least squares sense. The resulting factor for the data used was 1.25 , and the resulting comparison between modelled pressure in the cylinder at ivc and measured cylinder pressure at ivc is shown in Fig. 1. The correlation factor between estimated and measured pressure at ivc is 0.994 .

The total mass of different species in the cylinder at intake valve closing is calculated by adding the fresh air mass flow and the EGR mass flow. The fresh air mass flow is assumed to consist of $79 \% \mathrm{~N}_{2}$ and $21 \% \mathrm{O}_{2}$ (volume percentage). The composition of the species $\mathrm{N}_{2}, \mathrm{O}_{2}, \mathrm{CO}_{2}$, and $\mathrm{H}_{2} \mathrm{O}$ in the EGR flow is calculated by assuming equilibrium in the exhaust and intake manifolds, and by assuming that the injected fuel reacts perfectly with oxygen to create carbon dioxide and water according to:

$$
\mathrm{H}_{\alpha} \mathrm{C}+\left(\frac{\alpha}{4}+1\right) \mathrm{O}_{2} \rightarrow \mathrm{CO}_{2}+\frac{\alpha}{2} \mathrm{H}_{2} \mathrm{O}
$$

where:

$\alpha$ is the HC-ratio of the fuel (-) 


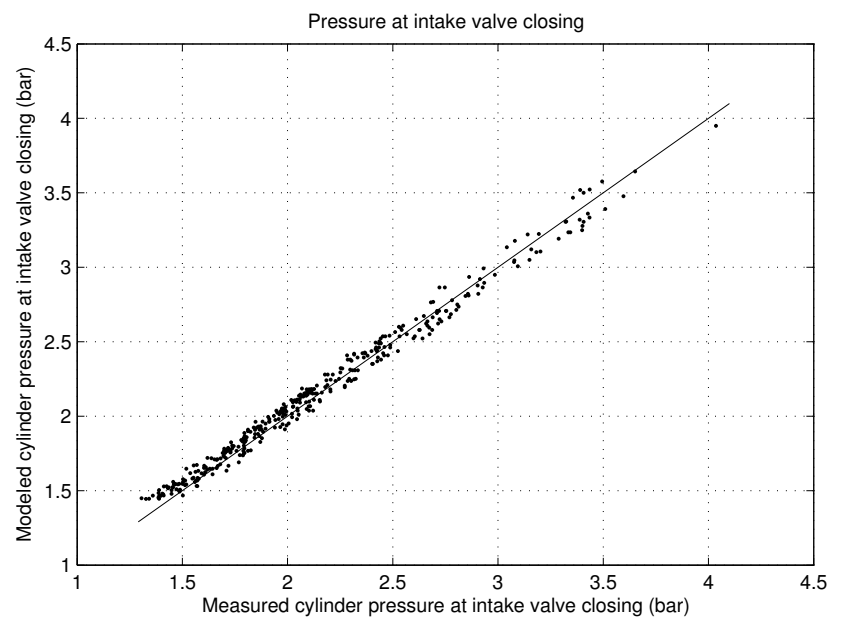

Fig. 1. Comparison between modelled and measured pressure in the cylinder at ivc for the data in the verification data set

The specific gas constant for the mixture in the cylinder at ivc, $R_{i v c}$, is directly calculated from the resulting gas composition in the cylinder. The cylinder volume at ivc, $V_{i v c}$, is calculated directly using the geometry of the engine, and the crank angle at intake valve closing (Heywood (1988)). Finally, the temperature in the cylinder at intake valve closing is calculated using the ideal gas law:

$$
T_{i v c}=\frac{p_{i v c} V_{i v c}}{m_{i v c} R_{i v c}}
$$

where:

$T_{i v c} \quad$ is the temperature in the cylinder at ivc $(K)$

$p_{i v c} \quad$ is the pressure in the cylinder at ivc $(\mathrm{Pa})$

$V_{i v c} \quad$ is the cylinder volume at ivc $\left(\mathrm{m}^{3}\right)$

$m_{i v c}$ is the mass in the cylinder at ivc $(\mathrm{kg})$

$R_{i v c}$ is the specific gas constant for the mixture in the cylinder at ivc $\left(\mathrm{Jkg}^{-1} \mathrm{~K}^{-1}\right)$

Using the estimated conditions in the cylinder at intake valve closing, the combustion process until the exhaust valve opening (evo) is estimated according to a simple model described in Egnell (1999).

$$
\frac{d Q}{d t}=C_{1}\left(Q_{f u e l}-Q\right)
$$

where:

$$
\begin{array}{ll}
d Q / d t & \text { is the heat release rate }(J / s) \\
Q & \text { is the accumulated heat release }(J) \\
Q_{f u e l} & \text { is the accumulated energy of the injected } \\
C_{1} & \text { fuel }(J)
\end{array}
$$

The accumulated energy of the injected fuel is expressed as:

$$
Q_{\text {fuel }}=Q_{L H V} \int_{t_{\alpha}}^{t} \dot{m}_{f u e l}(t) d t
$$

where:
$Q_{\text {fuel }}$ is the accumulated energy of the injected fuel $(J)$

$Q_{L H V}$ is the lower heating value of the fuel $(\mathrm{J} / \mathrm{kg})$

$\dot{m}_{f u e l} \quad$ is the fuel injection rate $(\mathrm{kg} / \mathrm{s})$

$t_{\alpha} \quad$ is the start of the injection $(s)$

$t \quad$ is the time of the calculation step in question $(s)$

The fuel injection rate, $\dot{m}_{f u e l}$, is estimated by a simple assumption that the fuel rate for one injection is constant, with start of injection at a constant time delay, $\tau_{\text {delay }}$, after the signal for the start of injection from the EMS, and with a duration directly calculated from the difference between the end of injection and start of injection from the EMS.

$$
\dot{m}_{\text {fuel }}=f\left(m_{i}, \text { soi }_{i}, \text { eoi }_{i}, \tau_{\text {delay }}\right)
$$

where:

$$
\begin{array}{ll}
m_{i} & \text { is the fuel amount in injection } i(\mathrm{~kg}) \\
s o i_{i} & \text { is the time for start of injection for injec- } \\
& \text { tion } i(\mathrm{~s})
\end{array}
$$

This means that the complete calculation from the fuel injection signal to the estimated rate of heat release profile is a model with two unknown calibration constants $\left(C_{1}\right.$ and $\left.\tau_{\text {delay }}\right)$. The calibration of these two constants was performed by calculating accumulated heat release profiles and accumulated fuel burn profiles using the cylinder pressure measurements. For the accumulated fuel burn profiles from the cylinder pressure measurements and from the model, the crank angles for when $10 \%, 50 \%$, and $90 \%$ of the fuel has been burned ( $m f b 10, m f b 50$ and $m f b 90)$ were calculated. The two calibration constants were calibrated such that the differences between measured and estimated angles for $m f b 10, m f b 50$, and $m f b 90$ were minimized in a least squares sense.

By performing this calibration individually for all different measured engine operating points, a strong engine speed dependency for the empirical constant $C_{1}$ was found. By adjusting the heat release model such that the heat release rate is based on the angular speed of the engine instead of time, the speed dependency was highly reduced. The adjusted heat release model could be described as:

$$
\frac{d Q}{d \theta}=C_{C A D}\left(Q_{f u e l}-Q\right)
$$

where:

$$
\begin{array}{ll}
d Q / d \theta & \text { is the heat release rate }(J / C A D) \\
Q & \text { is the accumulated heat release }(J) \\
Q_{f u e l} & \text { is the accumulated energy of the injected } \\
C_{C A D} & \text { fuel }(J)
\end{array}
$$

An explanation why this adjusted heat release model gives a better estimation of the heat release rate for the measurements could be that most of the fuel is combusted as a turbulent diffusion flame. The rate of combustion in a turbulent diffusion flame is limited by the degree of turbulence, and the degree of turbulence is typically higher for higher engine speeds (Heywood (1988)). 


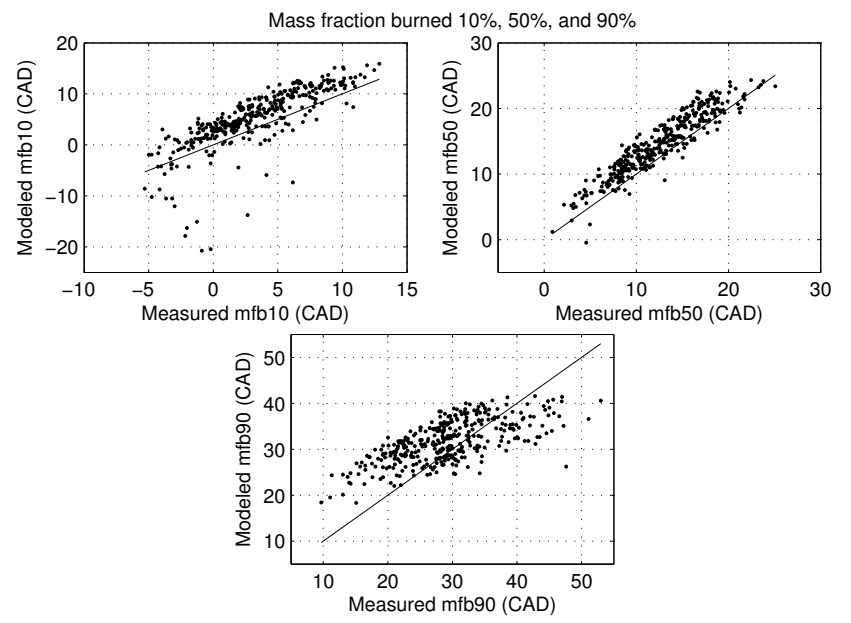

Fig. 2. Modelled crank angles for $m f b 10, m f b 50$, and $m f b 90$ versus crank angles calculated from the accumulated heat release obtained from the measured in-cylinder pressure for the verification data set

The optimized values for the two calibration parameters for the measurements were $\tau_{\text {delay }}=0.43 \mathrm{~ms}$ and $C_{C A D}=$ 0.074. The resulting comparison between $m f b 10, m f b 50$ and $m f b 90$ calculated from the measured cylinder pressure trace and calculated from the simulated fuel burn profiles are shown in Fig. 2. The correlation factor for the $m f b 10$ estimation is 0.781 , the correlation factor for the $m f b 50$ estimation is 0.928 , and the correlation factor for the $m f b 90$ estimation is 0.663 .

The estimation of $m f b 10$ is poor for some operating points. The main reason is that there is no model implemented for the ignition delay, which is closely connected to the position of $m f b 10$. The operating points with worst estimations are typically low load operating points, where the pilot injections sum up to more than $10 \%$ of the total injected fuel, but still are too small to ignite before the main injection. This is not captured in the combustion model, and result in large errors for the position of $m f b 10$ for those points.

Furthermore, the estimation of $m f b 90$, is, in general, a little bit worse than the estimations of $m f b 10$ and $m f b 50$. According to Chmela and Orthaber (1999), the injected fuel introduce kinetic energy into the cylinder which increase the turbulence, and therefore helps speeding up the fuel burn rate. However, the kinetic energy dissipates with time, and therefore the burn rate typically slows down towards the end of combustion, hence affecting the position of $m f b 90$. This phenomena is not considered in the model. Also, the slope of the accumulated heat release rate is lower towards the end of cumbustion, which directly leads to that the estimation of the position of $m f b 90$ becomes more sensitive than the estimations of $m f b 10$ and $m f b 50$.

A comparison of the estimated accumulated fuel burn profiles and accumulated fuel burn profiles calculated from the measured pressure trace for six different engine operating points are shown in Fig. 3. The operating points in the figure are all nominal operating point for the engine at $3000 \mathrm{rpm}$, with a total fuel injection of approximately

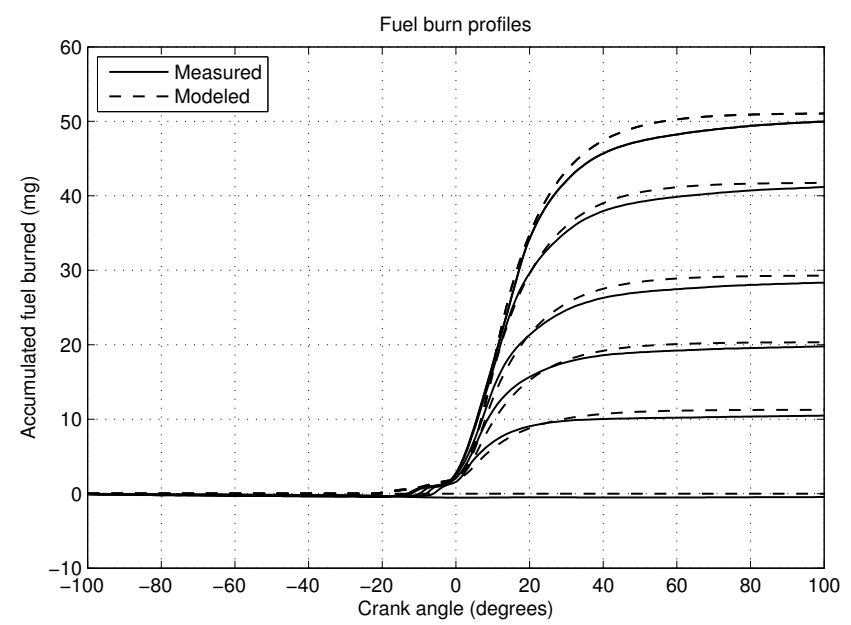

Fig. 3. Comparison between estimated accumulated fuel burn profiles and accumulated fuel burn profiles calculated from the measured in-cylinder pressure for six engine operating point from the verification data set

$0,10,20,30,40$, and $50 \mathrm{mg}$ per cylinder and cycle respectively.

The estimated fuel burn rate together with the concentration of different species before fuel injection starts is used to calculate the masses of the different species $N_{2}$, $\mathrm{O}_{2}, \mathrm{CO}_{2}$, and $\mathrm{H}_{2} \mathrm{O}$ throughout the complete combustion cycle using (2).

The heat release rate together with the concentration of different species throughout the combustion is used to create a simulated cylinder pressure trace from intake valve closing to exhaust valve opening according to Heywood (1988), neglecting the crevice effects and the mass change due to the fuel injection.

$$
\delta Q=\left(\frac{c_{v}}{R}\right) V d p+\left(\frac{c_{v}}{R}+1\right) p d V+\delta Q_{h t}
$$

where:

$\delta Q \quad$ is the heat release $(J)$

$c_{v} \quad$ is the specific heat capacity at constant volume $\left(J k g^{-1} K^{-1}\right)$

$R \quad$ is the specific gas constant $\left(\mathrm{Jkg}^{-1} \mathrm{~K}^{-1}\right)$

$V \quad$ is the cylinder volume $\left(\mathrm{m}^{3}\right)$

$d V \quad$ is the cylinder volume change $\left(\mathrm{m}^{3}\right)$

$p \quad$ is the cylinder pressure $(P a)$

$d p \quad$ is the cylinder pressure change $(\mathrm{Pa})$

$T$ is the temperature $(K)$

$Q_{h t} \quad$ is the heat transfer to the cylinder walls $(J)$

The specific heat capacity, $c_{v}$, is calculated throughout the combustion using NASA polynomials (Burcat and Ruscic (2005)) for the different species and the temperature given by the ideal gas law. The specific gas constant, $R$, is calculated from the known concentration of different species throughout the combustion. The heat transfer to the cylinder walls is calculated using Woschni's correlation as described in Heywood (1988).

Finally, to achieve a simulated pressure trace for the complete combustion cycle, the cylinder pressure from the piston bottom dead centre (BDC) to before the intake valve closing is set to the pressure in the intake manifold, with 


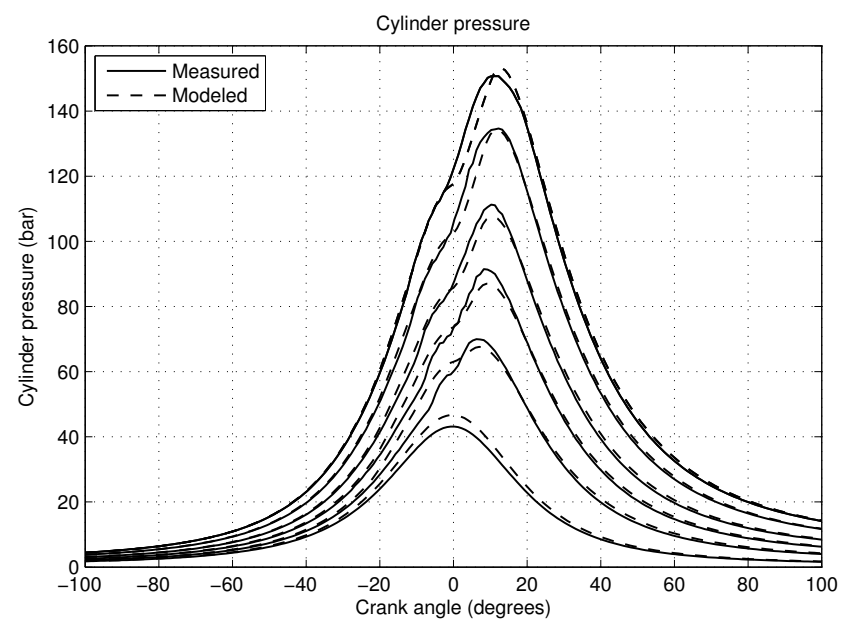

Fig. 4. Estimated cylinder pressure traces compared with measured cylinder pressure traces for six different engine operating points taken from the verification data set

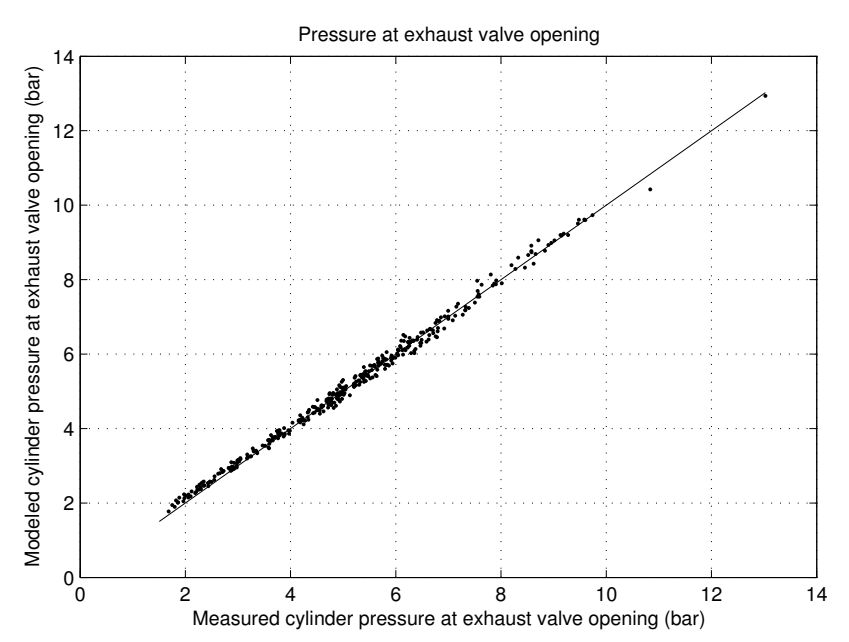

Fig. 5. Estimated pressure compared to measured pressure in the cylinder before exhaust valve opening for the engine operating points in the verification data set

linear interpolation to the calculated cylinder pressure at intake valve closing. Similarly, the cylinder pressure from the exhaust valve opening to $\mathrm{BDC}$ before the intake valve opening is set to the pressure in the exhaust manifold.

A comparison between measured and simulated cylinder pressure for the six different engine operating points is shown in Fig. 4. The operating points are the same operating points as shown in Fig. 3, i.e. six nominal engine operating points at $3000 \mathrm{rpm}$. A comparison of the estimated and measured pressure before exhaust valve opening is shown in Fig. 5. The correlation factor for the estimated cylinder pressure before exhaust valve opening is 0.998 .

Using the simulated pressure in the cylinder, an estimated value for $I M E P_{n e t}$ is calculated. A comparison between $I M E P_{n e t}$ calculated from measured cylinder pressure traces versus simulated cylinder pressure is shown in Fig. 6. The correlation factor for the $I M E P_{n e t}$ estimation is 0.995 .

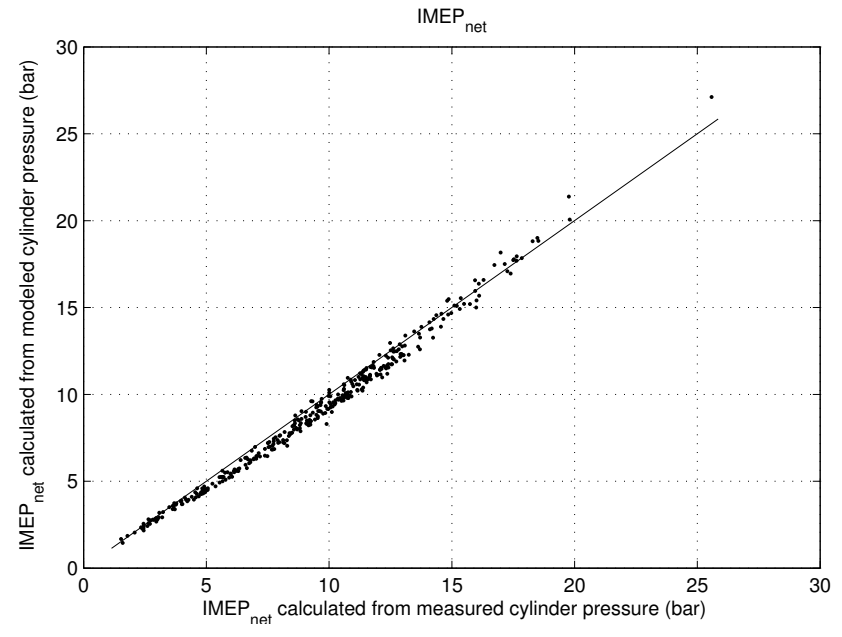

Fig. 6. $I M E P_{\text {net }}$ calculated from the estimated cylinder pressure traces versus $I M E P_{n e t}$ calculated from measured cylinder pressure traces for the engine operating points in the verification data set

The complete combustion model takes approximately 25 ms to evaluate for one combustion cycle with a crank angle resolution of $1 \mathrm{CAD}$ on a standard computer. Using matrix calculations in Matlab, several operating points can be simulated simultaneously, decreasing simulation time to approximately $1 \mathrm{~ms}$ per cycle.

\subsection{Mean value model coupling}

The combustion model has been coupled to a mean value model of an engine air system. The air system model is based on a model described in Wahlström (2009), but to be able to provide the combustion model with the desired prerequisites, the model has been updated to include states for the masses of the different species $\mathrm{O}_{2}, \mathrm{~N}_{2}, \mathrm{CO}_{2}$, and $\mathrm{H}_{2} \mathrm{O}$ in the intake and exhaust manifolds. The mean value model has continuous states, while the combustion model is a static model which simulates one combustion cycle using conditions in the manifolds. Therefore, these two models cannot be connected directly. The approach taken to solve this problem is:

The mass flows into the engine in the mean value model are continuously accumulated:

$$
m_{i n, i}=\int \dot{m}_{i n, i} d t
$$

where:

$m_{i n, i}$ is the accumulated mass of species $i$ in to the combustion chamber $(\mathrm{kg})$

$\dot{m}_{i n, i}$ is the mass flow of species $i$ in to the combustion chamber $(\mathrm{kg} / \mathrm{s})$

From the continuous signal for the engine speed, a trigger signal is produced with intervals matching the intervals between the combustions in the cylinders, i.e., there is a trigger signal every $2 / n_{c y l}$ engine revolution, where $n_{c y l}$ is the number of cylinders in the engine. The trigger signal activates one calculation of the combustion process. The combustion model uses the pressure in the intake manifold and the pressure in the exhaust manifold at the time of the trigger signal, together with the accumulated 
masses of different species as inputs for its calculations. The trigger also resets the accumulation of masses, i.e. it sets $m_{i n, i}=0$.

The calculation of the combustion process produces an estimated cylinder pressure trace for the complete combustion cycle, and it also produces the total mass of different species at the time when the exhaust valves open. From the produced masses in the cylinder, mass flows out from the cylinder to the exhaust manifold needs to be created such that the accumulated mass flows equal the produced masses exactly at the time of the following trigger signal. This is achieved by continuously predicting the remaining time to next trigger signal, using the derivative of the engine speed signal. By dividing the remaining fuel masses with the predicted remaining time, continuous mass flows out from the combustion chamber to the exhaust manifold are created. This means that the air system part of the model experience the combustion part of the model as a volumetric pump, the same way as described in Wahlström (2009), while the combustion model is executed as discrete events, with given boundary conditions.

\section{DISCUSSION}

The combustion model that has been implemented is fast to execute, and it reacts on the controllable parameters boost pressure, exhaust gas recirculation rate, and the injection strategy with timings and amounts for different injections. The coupling with a mean value model of the air system also enables simulation of transient engine behaviour, capturing air system dynamics. The coupling also leads to that changes in the controllable engine parameters affect the complete engine system more than just by the direct effects. For example, the injection strategy for one combustion cycle do not only effect the engine performance on that particular cycle, it also affects the temperature and pressure in the exhaust manifold, which in turn affect the energy available for the turbocharger and so forth. This means that it is possible to use the model to develop engine calibration optimizing strategies that accounts for these effects.

The model is based on physical relations, with a few added empirical parameters. This implies that the model should be able to produce reasonable estimations also for engine operating points outside the range of the operating points used for the calibration of the model. This is a desirable feature for several reasons. If dynamic engine behaviour is to be simulated, the conditions in the intake and exhaust manifolds during transients may include states that are not possible to reach in steady-state engine operation, unless using a research engine, e.g. a single-cylinder engine with possibility to independently control the pressure at the exhaust side of the engine. It also opens up the possibility to study how changes in the air system, for example a resized turbo charger can influence the engine operation without the need of new calibration measurements.

The simplicity of the combustion model makes it very fast to execute, which is a nice property for optimization using the model. However, if higher degree of accuracy is needed, the model could be extended to be more detailed, for example by adding a model for the ignition delay as described in Egnell (1999), a model for the kinetic energy induced by the injections as described in Chmela and Orthaber (1999), or a model for residual mass within the cylinder (Heywood (1988)). The fast execution time of the model also means that the model, with minor alterations, should be possible to implement into the EMS of a real engine.

The authors intended usage of the model is to couple it with fast models for particulate matters $(P M)$ and $N O_{x}$ emissions, and thereby be able to simulate dynamic engine operation while getting estimations on both fuel consumption and emissions, depending on the controllable engine parameters. Doing this, optimization strategies for the EMS will be studied, where the target is to optimize the EMS to minimize fuel consumption during a specified dynamic cycle, while fulfilling requirements on accumulated $P M$ and $N O_{x}$ emissions.

\section{REFERENCES}

Andersson, M., Johansson, B., Hultqvist, A., and Nöhre, C. (2006). A real time nox model for conventional and partially premixed diesel combustion. SAE Paper 200601-0195.

Arsie, I., Pianese, C., and Sorrentino, M. (2007). Control parameters optimization in automotive diesel engines via two zone modelling. Advances in Automotive Control, 5(1).

Atkinson, C., Allain, M., and Zhang, H. (2008). Using model-based rapid transient calibration to reduce fuel consumption and emissions in diesel engines. SAE Paper 2008-01-1365.

Brahma, I. and Rutland, C. (2003). Optimization of diesel engine operating parameters using neural networks. SAE Paper 2003-01-3228.

Brahma, I., Sharp, M., and Frazier, T. (2009). Empirical modeling of transient emissions and transient response for transient optimization. SAE Paper 2009-01-1508.

Burcat, A. and Ruscic, B. (2005). Third millennium ideal gas and condensed phase thermochemical database for combustion with updates from active thermochemical tables. Technical Report ANL-05/20 and TAE 960, Technion-IIT, Aerospace Engineering and Argonne National Laboratory, Chemistry Division.

Chmela, F.G. and Orthaber, G.C. (1999). Rate of heat release prediction for direct injection diesel engines based on purely mixing controlled combustion. $S A E$ Paper 1999-01-0186.

Egnell, R. (1999). A simple approach to studying the relation between fuel rate, heat release rate and no formation in diesel engines. SAE Paper 1999-01-3548.

Heywood, J.B. (1988). Internal Combustion Engine Fundamentals. McGraw-Hill.

Kirchen, P. and Boulouchos, K. (2009). Development and validation of a phenomenological mean value soot model for common-rail diesel engines. SAE Paper 2009-01$127 \%$.

Shrivastava, R., Hessel, R., and Reitz, R. (2002). Cfd optimization of di diesel engine performance and emissions using variable intake valve actuation with boost pressure, egr and multiple injections. SAE Paper 200201-0959.

Wahlström, J. (2009). Control of EGR and VGT for Emission Control and Pumping Work Minimization in Diesel Engines. Ph.D. thesis, Linköping University. 